\title{
Field-Safety Notice and Recall of Sleep Care Devices by Philips Respironics
}

\author{
Deepak Shrivastava ${ }^{1} \cdot$ Velayudhan Mohan $\operatorname{Kumar}^{2} \cdot$ Ravi Gupta $^{3} \cdot$ Hruda Nanda Mallick $^{4}$
}

Published online: 20 July 2021

(c) The Author(s), under exclusive licence to Springer Nature Singapore Pte Ltd. 2021

\begin{abstract}
On 14th June 2021, Philips Respironics issued a medical device recall notification (US only) and field-safety notice (International Markets) for mechanical ventilator devices including continuous positive airway pressure (CPAP), and bilevel positive airway pressure (bilevel PAP). This was in response to potential health risks related to the sound abatement foam component in these devices. The company had stated that they are working hard towards a resolution, which includes repair and replacement of the affected devices. The statement of Philips clearly declares that this is a recall notification for the US, and only a field-safety notice for the rest of the world. The advisory from Philips puts the ball in the court of the physician or Durable Medical Equipment (DME) provider to determine the most appropriate options for continued treatment of patients using the affected devices. Although the long-term effects of these recalled machines will continue to be a matter of concern for the patients, the patients must be supported by the providers with explanations, reassurances and hope.
\end{abstract}

\section{Introduction}

The sleep industry round the world is going through a pandemonium, reacting to the recent recall of certain sleep and respiratory care devices by Philips Respironics. As a result, the suppliers, vendors and the durable medical equipment (DME) companies are in a state of panic. Patients are confused and scared and nobody is sure about the potential solutions. Philips Respironics advised the affected patients to contact their physicians. The World Sleep Society has created a webpage for gathering information from societies and other organizations regarding this notice $[1,2]$. There are many questions physicians worldwide have to answer to the voluntary medical device recall notification in the US, and to the field-safety notice to non-US markets by Royal Philips.

Hruda Nanda Mallick

drhmallick@yahoo.com

1 Division of Pulmonary, Critical Care and Sleep, Department of Medicine and Medical Director, SJGH Sleep Center, UC Davis School of Medicine, Sacramento, CA, USA

2 Kerala Chapter, National Academy of Medical Sciences (India), New Delhi, India

3 Department of Psychiatry and Division of Sleep Medicine, All India Institute of Medical Sciences, Rishikesh, India

4 Faculty of Medicine and Health Sciences, SGT University, Budhera, Gurugram, India

\section{Genesis of the Problem}

On April 26, 2021, Philips announced its financial results in the first quarter of 2021, and reported a EUR 250 million provision for "a quality issue in a component that is used in certain sleep and respiratory care products." The company specifically revealed "possible risks to users related to the sound abatement foam used in certain of Philips' sleep and respiratory care devices currently in use" [3]. On 14th June 2021, Philips Respironics issued a medical device recall notification (US only) and field-safety notice (International Markets) for mechanical ventilator devices [4]. According to Philips Respironics, the list of the affected devices includes continuous positive airway pressure (CPAP), bilevel positive airway pressure (BiPAP), bilevel positive airway pressureST mode (BiPAP-ST) and Trilogy 100/200 ventilators. Of the approximately 4 million devices affected by the recall, $80 \%$ of them are CPAP machines and its variants; other $20 \%$ are the ventilators [5].

\section{Root Cause of the Problem}

The recall/field-safety notice is related to the type of foam used to reduce the noise made by the devices. Over time, the foam inside the machine may have disintegrated into black particles. These particles can enter the humidifier, 
tubing and mask. As a result, one may inhale these particles when using the device. Philips reports that the potential risks include headache, skin or eye irritation and asthma [6]. Tests conducted by Philips also found that the foam can produce unsafe chemical levels. These "volatile organic compounds" (VOCs) are released as gases. Exposure to VOCs may cause problems such as irritation of the airway, headache or dizziness, skin, eye or nose irritation and nausea or vomiting. Philips reports that in 2020 the complaint rate for foam particles was low $(0.03 \%)$. In the statement, under the possible health risks, Philips includes toxic and carcinogenic effects, apart from the above-mentioned problems. Philips states that it has received no complaints related to chemical exposure. They also stated that there have been no reports of death.

\section{Global Impact}

On behalf of the company, Frans van Houten, CEO of Royal Philips issued a statement in the global press, "We deeply regret any concern and inconvenience that patients using the affected devices will experience because of the proactive measures, we are announcing today to ensure patient safety. In consultation with the relevant regulatory agencies and in close collaboration with our customers and partners, we are working hard towards a resolution, which includes the deployment of the updated instructions for use and a comprehensive repair and replacement program for the affected devices" [7]. An industry source confirmed to Business Today (India) "Following the recall notification issued by Philips in the US market, the Dutch medical equipment company will voluntarily recall impacted machines, including breathing devices and ventilators in India" [8]. They stated that the customers can also call its helpline number 18002587678 for support and more information.

\section{Initiatives from Philips}

In spite of the above information published in Business Today (India), the notification of Philips issued on 14th June 2021 clearly states that this is a recall notification for the US only, and a field-safety notice for the rest of the world. It states the following:

- Philips is initiating a voluntary recall notification to ensure patient safety in consultation with regulatory agencies.

- Corrective actions include the deployment of updated instructions for use and a repair and replacement program for affected devices.

- Philips aims to address all affected devices within the scope of this correction as expeditiously as possible.
It may be noted that the statement makes no indication of the time frame of repair and replacement program for affected devices, even in the US.

\section{Advise from Philips}

The recall notification gives two different advises for patients and customers.

- For patients using affected BiLevel PAP and CPAP, it advises discontinuation of the device and work with their physician or DME provider to determine the most appropriate options for continued treatment, and for continued use of device due to lack of alternatives.

- For patients using affected life-sustaining mechanical ventilator, it advises not to stop or alter the prescribed therapy until they have talked to their physician.

Essentially the advisory puts the ball in the court of the physician or DME provider to determine the most appropriate options for continued treatment. Though the physicians are certainly capable of giving appropriate advice, it puts them in a situation where they are answerable for consequences for which they are not responsible. They would be also answerable for any adverse consequences which may happen during continued use of the devices. Technology disasters are common and most of them are promptly rectified. As stated earlier, approximately 4 million devices were affected by the recall, and $80 \%$ of them are CPAP machines [5]. Therefore, this technology disaster has worldwide implications on the manufacturing and shipment of the CPAP, BiPAP and home ventilators for the foreseeable future.

\section{Options Before the Sleep Specialists [5]}

(1) At this point, it is important to recognize that there may be patients who are dependent on these ventilators and will not survive without the assisted mechanical ventilation.

(2) A second group of patients are those who suffer from obstructive sleep apnea with many comorbid conditions and are at significant risk of an adverse event due to the nocturnal hypoxemia and hypoventilation without the use of their positive airway pressure machine.

(3) A third group can be identified as the patients with obstructive sleep apnea but without significant comorbid conditions.

Patients requiring a ventilator for life-sustaining therapy can consider switching over to ResMed and Fisher and Paykel healthcare products. Both companies have risen to 
the occasion and are getting more engaged with the affected patient population. Given the current situation, the newly diagnosed patients with obstructive sleep apnea may be tempted to choose ResMed and Fisher and Paykel products. It may be noted that Fisher and Paykel healthcare companies sells their CPAP devices in the private pay market in the US. While it might take some time to get the preauthorization from the third-party payer and insurance companies, the patient's choices may be limited to continuing with the recalled ventilator.

Should the patient decide to continue using the recalled ventilator, according to Philips Respironics, they should not use ozone-related cleaning products, and adhere to their device instruction for approved cleaning methods. Philips Respironics also recommends that patients review the age of their BiPAP and CPAP, as it is recommended that these devices are to be replaced after five years of use. Philips Respironics recommends addition of an in-line bacterial filter, if the machine has to be used continuously while alternative arrangements are made.

The second and third group of patients who use CPAP or AutoPAP machines for mild to moderate sleep apnea, according to current recommendation, may stop using the machine at their discretion but follow standard supporting interventions:

(1) Not sleeping on the back or place a pillow behind the back to prevent from rolling over during the sleep. Many people have higher incidence of abnormal breathing events in supine position due to gravitational force.

(2) Avoid alcohol, sedatives or narcotics close to the bedtime. These substances are known to impair muscle tone and increase the incidence of abnormal breathing events.

(3) Discuss with the provider alternative options such as permanent or temporary oral appliances, supplemental oxygen and a variety of surgical interventions.

\section{Unanswered Issues}

This is a welcome step from an industry giant, where they have opted for taking the corrective options in view of the available information, rather than sweeping it under the carpet. However, the data regarding the details of medical problems that the patients who used the affected devices have not been made available to the scientific community. It is also surprising that this issue has not appeared in any of the medical journals so far, yet it came to the notice of the manufacturer. Physicians are dealing with the patients directly and are morally, ethically and legally responsible for their welfare. Philips is expected to share the detailed information related to the following issues so that appropriate management steps can be taken by the physicians:

(1) How did it come to the notice of Philips?

(2) When did it first come to the notice of Philips?

(3) Was it reported by the physicians who treated the patients?

(4) Was it reported by the patients? If yes, were the physicians made aware of the issue?

(5) Which group of patients was most affected?

(6) What was the magnitude of the symptoms?

(7) How did Philips arrive at the figure of $0.03 \%$ ?

(8) Was it just an association or has the causal link been established?

(9) What would happen to the patients using PAP devices in countries like India, where they have purchased these devices by shelling out money from their own pockets?

Moreover, due to lack regulatory rules and gaps in the structuring of the health-care system, in many developing countries, large numbers of PAP devices are prescribed by the distributors of these devices rather than by the physicians.

\section{Concluding Remarks}

Philips Respironics recommends registering the affected machines on their website. The repair service and cost of shipping is free within the US (Please note that for international patients, the repair is free; however, the shipping is not covered by Philips Respironics).

Philips Respironics Dream Station, the most affected device directly competes with the ResMed air sense 10 . If the machine is 5 years or older, it can be substituted with the approval of the insurance company immediately.

One should be reminded, however, that these devices have been in use for past 5 years and no significant health-related problems have been reported, other than the ones mentioned earlier.

Although, long-term effects related to potential carcinogenesis will continue to be a matter of concern for few patients who have used these recalled machines. Meanwhile, patients must be supported by the providers with explanations, reassurances, and hope.

\section{References}

1. World Sleep Society News: Philips Issues Field Safety Notice for Certain Sleep and Respiratory Care Devices. https://worldsleep society.org/philips-field-safety-notice/. Accessed 22 June 2021. 
2. ISSR News: Field-safety notice by Royal Philips. (ISSR website). https://issr.in/wp-content/uploads/2021/06/Field-safety-notice-byRoyal-Philips.pdf. Accessed 26 June 2021.

3. The Law Offices of Frank R. Cruz Announces Investigation of Koninklijke Philips N.V. (PHG) on Behalf of Investors. https:// www.barrons.com/press-release/the-law-offices-of-frank-r-cruzannounces-investigation-of-koninklijke-philips-n-v-phg-onbehalf-of-investors-01623857413?tesla $=y \&$ tesla $=y$. Accessed 22 June 2021.

4. Medical Device recall notification (U.S. only) / field safety notice (International Markets). https://www.usa.philips.com/healt hcare/e/sleep/communications/src-update. Accessed 23 June 2021.

5. Shrivastava D. The Ventilator Crisis, Panic Mode and Sensibility. https://www.bestsleepdoctor.com/public/The-Ventilator-CrisisPanic-Mode-and-Sensibility.cfm. Accessed 22 June 2021.

6. Philips PAP device recall: Guidance for patients. https://sleep education.org/philips-pap-device-recall-guidance-for-patients/. Accessed 23 June 2021.
7. Philips provides update on recall notification - News I Philips. https://www.philips.com/a-w/about/news/archive/standard/news/ press/2021/20210614-philips-issues-recall-notification-to-mitig ate-potential-health-risks-related-to-the-sound-abatement-foamcomponent-in-certain-sleep-and-respiratory-care-devices.html. Accessed 22 June 2021.

8. Philips India to recall faulty breathing devices, mechanical ventilators over safety. https://www.businesstoday.in/current/corporate/ philips-to-recall-faulty-breathing-devices-ventilators-in-indiaover-health-safety-issues/story/441802.html. Accessed 22 June 2021.

Publisher's Note Springer Nature remains neutral with regard to jurisdictional claims in published maps and institutional affiliations. 